\title{
A Dynamic Mathematical Modeling Revelation about the Impact of Vaccination on Hepatitis B Virus-induced Infection and Death Rate in Bangladesh
}

\author{
Sajib Chakraborty ${ }^{1}$, Rajib Chakravorty ${ }^{2}$, Saruar Alam ${ }^{3}$, Yearul Kabir ${ }^{4}$, Musarrat Mahtab ${ }^{5}$, Md Atikul Islam ${ }^{6}$ \\ Md Abul Khair Yusuf ${ }^{7}$, Ruksana Raihan ${ }^{8}$, Mamun Al Mahtab ${ }^{9}$, Sheikh Mohammad Fazle Akbar ${ }^{10}$
}

\begin{abstract}
Aim: Attainment of sustainable development goal (SDG) targets requires reducing the rate of new hepatitis B virus (HBV)-induced infection and mortality rate to $90 \%$ and $65 \%$, respectively, by 2030 . Therefore, it is important to investigate the feasibility of reducing the required rates of $\mathrm{HBV}$-induced infection and death incidents at the current rate of vaccination coverage in Bangladesh. Moreover, factors influencing vaccination coverage like negative bias toward girls during immunization can affect the current vaccination program and ultimately hinder the efforts to reduce $\mathrm{HBV}$-induced infection and death rates. To investigate the possibility of reducing HBV-induced infection and death rates with current vaccination coverage, we adopted mathematical molding-based approach.

Materials and methods: We developed a mathematical model based on the susceptible-infectious-recovered model to simulate the HBV-induced infection in children under the age of five at three different vaccination rates: 80,90 , and $95 \%$. Additionally the impact of current vaccination coverage was assessed on HBV-induced death rates in the future. Moreover, we took advantage of the mathematical model to investigate the impact of negative bias toward girls in vaccination program on HBV-induced infection and death rates.

Results: The model simulations revealed that $10 \%$ increase in the vaccination rate from 80 to $90 \%$ can potentially contribute to the significant lowering (around $40 \%$ ) of HBV-induced infection rate among children. When increased by $5 \%$ of vaccination rate from 90 to $95 \%$, the HBV-infection rate is likely to be decreased by another $22 \%$. Likewise, $44 \%$ reduction in HBV-induced death rate in the future (2050 onward) can potentially be achieved by $10 \%$ increase in the current vaccination rate from 80 to $90 \%$, whereas $5 \%$ increase in the current vaccination rate ( $90-95 \%$ ) may lead to $24 \%$ further reduction of death rate. These results underscored the significant impact of vaccination in reducing HBV-induced infection among children and future death rates in adults. Moreover, at $90 \%$ vaccination coverage, the negative bias of vaccination toward girls contributes to an increase of 15 and $12 \%$ of HBV-induced infection and death rates, respectively, in female subjects compared to their male counterparts.

Conclusion: The current vaccination coverage (80-90\%) is further aggravated by untimely vaccination, dropouts from vaccination program, and negative bias toward girls in vaccination program. Therefore, if the current situation persists, it will not be possible to accomplish the required reduction in HBV-induced infection and death rates by 2030 , according to the SDG guidelines. Moreover negative bias in the vaccination program may intensify the HBV-induced infection and death rates in the future.

Clinical significance: In light of the mathematical model, we suggest that the vaccination coverage should be increased to $95 \%$ without any negative bias toward girls. To accomplish this, the concerning authorities must ensure timely and full completion of the HBV vaccine schedules, reducing dropouts from vaccination program, and lastly preventing negative bias toward girls to uplift vaccination coverage to more than $95 \%$ with gender equality. Without these strategies, the necessary reduction in the HBV-induced infection and death rates in Bangladesh may not be attained per SDG directives. Keywords: Hepatitis B virus, Immunization, Mathematical model, Target.

Euroasian Journal of Hepato-Gastroenterology (2019): 10.5005/jp-journals-10018-1303
\end{abstract}

\section{INTRODUCTION}

Hepatitis $B$ virus infection is the leading cause of a wide-spectrum of liver disease such as chronic hepatitis, liver cirrhosis, and hepatocellular carcinoma (HCC). ${ }^{1}$ According to the World Health Organization (WHO), nearly 257 million people are living with HBV infection globally and HBV infection was held responsible for 8,87,000 deaths in 2015 (https://www.who.int/news-room/factsheets/detail/hepatitis-b). The prevalence rate of HBV varies with different geographical location, where the highest prevalence of HBV infection has been reported in African regions (8.83\%) and Western Pacific region (5.26\%). ${ }^{2,3}$ In Bangladesh, the large-scale epidemiological data regarding the population-wide prevalence of HBV infection and death rates are lacking. ${ }^{4}$ Although few studies attempted to uncover the HBV infection prevalence in Bangladesh, the estimated prevalence was variable among these studies. For instance, a study by Mahtab et al. including 1,018 individuals reported that the crude prevalence of $\mathrm{HBV}$ infection is around $5.5 \%$ in Bangladesh. ${ }^{5}$ Whereas Schweitzer et al. compiled the data from

\begin{abstract}
1,3,4 Department of Biochemistry and Molecular Biology, Translational Systems Biology Laboratory, University of Dhaka, Bangladesh

${ }^{2}$ Department of Applied and Photonics, University of Sydney, Sydney, Australia

${ }^{5}$ Forum for the Study of the Liver, Dhaka, Bangladesh

6,7,9 Department of Hepatology, Bangabandhu Sheikh Mujib Medical University, Dhaka, Bangladesh

${ }^{8}$ Department of Medicine, University of Malaya, Kuala Lumpur, Malaysia ${ }^{10}$ Department of Pathology, Proteo-Science Center, Ehime University Graduate School of Medicine, Ehime, Japan

Address reprint requests to: Sajib Chakraborty, Department of Biochemistry and Molecular Biology, Translational Systems Biology Laboratory, University of Dhaka, Bangladesh, Phone: +880-29661900/7636, e-mail: sajib@du.ac.bd

How to cite this article: Chakraborty S, Chakravorty R, Alam S, et al. A Dynamic Mathematical Modeling Revelation about the Impact of Vaccination on Hepatitis B Virus-induced Infection and Death Rate in Bangladesh. Euroasian J Hepato-Gastroenterol 2019;9(2):84-90.
\end{abstract}


16 studies covering 94,352 participants and estimated the HBV prevalence as $3.10 \%{ }^{2}$

Transmission of HBV typically occurs through the exposure to infected blood and bodily fluids. The major routes of HBV transmission include sexual intercourse, parenteral contact, or HBV exposure in the newborn at birth from an HBV-infected mother. ${ }^{6}$ Growing body of evidence suggests that in the developing countries the most frequent infection route is mother-to-child vertical transmission and horizontal transmission between children, especially among siblings. ${ }^{7}$ Vertical transmission from mother to child has emerged as a great threat since the risk of developing chronic HBV $(\mathrm{CHB})$ infection among infants during the first year after vertical transmission at birth is as high as $90 \%$ and the risk is reduced to $30-50 \%$ when the age is below 6 years during the time of infection. ${ }^{8}$ Epidemiological studies established a direct link between $\mathrm{CHB}$ infection and the development of a variety of liver diseases including $\mathrm{HCC}^{9}$ which ultimately leads to death. The CHB-infected (CHB) patients were more susceptible to HBV-induced deaths than their noninfected counterparts.

The $\mathrm{CHB}$ patients were subjected to death at an average age of 59.8 years, which is 14 years earlier than the general population. ${ }^{10}$ Another study showed that death rates due to liver abnormality in $\mathrm{CHB}$ subjects are significantly higher after 40 years of age. ${ }^{11}$

The HBV vaccination program has been introduced since 1980 and has proved to be effective to boost long-lasting immunity against $\mathrm{HBV}$ and thus was considered as highly successful in reducing $\mathrm{HBV}$-associated disease burden. ${ }^{12}$ Realizing the importance of HBV vaccination, WHO recommended the inclusion of $\mathrm{HBV}$ vaccination at birth in the national immunization programs worldwide. ${ }^{13}$ In Bangladesh, the inclusion of HBV vaccine into the expanded program on immunization (EPI) was materialized in different phases during 2003 to 2005 following the WHO recommended vaccination schedules at 6,10 , and 14 weeks of age. ${ }^{14}$ In Bangladesh, several socioeconomic factors have been identified to influence the childhood vaccination rate. For instance, children born to mothers with low educational backgrounds and in the lowest wealth quintile are less likely to be fully vaccinated due to the less frequent visits to vaccination care units. ${ }^{15}$ As a consequence, full vaccination coverage remains elusive in Bangladesh. In 2014, 83\% vaccination coverage was attained in Bangladesh. ${ }^{16}$ In Bangladesh, another challenge in the EPI program is negative biases against girls and, consequently, female children have lower immunization coverage compared to their male counterparts. In Bangladesh, according to Global Alliance for Vaccines and Immunization (GAVI) alliance report (https://www.odi.org/sites/ odi.org.uk/files/odi-assets/publications-opinion-files/5315.pdf).

One of the SDGs is to ensure healthy lives and promote well-being for all age-groups (https://www.un.org/sustainabledevelopment/ health/). Under this goal, a target is set to reduce the new incidence of CHB infection and HBV-induced mortality rate by 90 and $65 \%$, respectively, by the 2030 (http://apps.who.int/gb/ebwha/pdf_files/ WHA69/A69_32-en.pdf?ua=1). However, the question remains, regarding the current rate of vaccination coverage, and the existing socioeconomic challenge is whether Bangladesh can realistically achieve the SDG requirements of reducing HBV-induced infection and death rates. Moreover, how the factors influencing vaccination coverage such as incomplete vaccination, vaccination-schedule miss, dropouts from vaccination program, and negative biases against girls during immunizations will affect the current vaccination coverage and ultimately hinder our efforts to reduce HBV-induced infection and death rates are unclear. Mathematical models especially "susceptible-infectious-recovered" commonly known as SIR model has been proved to be the most effective in capturing the realistic

\section{Source of support: Nil}

Conflict of interest: None

epidemiological pattern of infectious diseases and assessing the impact of vaccination on the spread of diseases. ${ }^{17}$ To address the crucial question, whether Bangladesh can accomplish the required reduction in HBV-induced infection and death rates per SDG requirements, we adopted mathematical molding-based approach where we have modified the SIR model to simulate the HBV-induced infection and death rates in children under the age of 5 at different vaccination rates ranging from 80 to $95 \%$. Moreover, we used the modified SIR model to investigate the impact of negative biases against girls in vaccination program on HBV-induced infection and death rates.

\section{Materials and Methods}

\section{Model Description}

Among the mathematical models that are currently available to study epidemiology, SIR is considered to be the most popular and paradigmatic model in epidemiology where the transition of an individual is unidirectional from susceptible to recovered compartment after subsiding the infection. ${ }^{18}$ We modified SIR model to evaluate the impact of vaccination on infection and death rate due to HVB in Bangladeshi children. Typically the SIR model is formulated based on a set of differential equations that facilitate the modeling of the infection and death rates over time deterministically. In SIR model, the population is divided into three compartments, namely, susceptible (S), infectious (I), and recovery $(R)$, where an individual can be in any of the three states at a given time point. Transition can occur between compartments in a unidirectional manner when the fraction of a population of susceptible compartment becomes infected or the fraction of a population of infected individual recovers. The total population size $N$ is the sum of population in all compartments $(S+I+R)$. Typically the total population size $(N)$ is fixed, but the population size of $S, I$, and $R$ compartments are subjected to change with time, according to the equations. However, a fixed total population size $(N)$ is unrealistic simply because it does not account for demographic changes such as live birth and death rates. Birth and death rate of a population can have significant impact on the infection and death rates for a particular infectious disease. Previously a term- "buffering" has been introduced as a measure of a sensitivity of the dynamics of an infectious disease with respect to changes in demographic parameters such as birth and death rates. ${ }^{19}$ Therefore, to align the model close to reality, we took into consideration the live birth and death rates of Bangladesh. Apart from $S, I$, and $R$ compartments, we have introduced another compartment that is denoted as healthy $(\mathrm{H})$. We defined $\mathrm{H}$ compartment as the fraction of the total population that are not susceptible, infected, or recovered at a given point of time. The population of the $\mathrm{H}$ compartment is those healthy individual that are not susceptible to HBV infection and are not unlikely to be infected in the future. The reason behind introducing this additional compartment $(\mathrm{H})$ is to differentiate between nonsusceptible and susceptible individuals. For example, the children born to HBVinfected mothers are more susceptible compared to the newborns of uninfected mothers. The HBV infection may occur during birth, infancy, and early childhood, mostly through the mother-tochild vertical transmission in HBV-endemic areas, accounting for approximately half of the transmission routes of $\mathrm{CHB}$ infections. ${ }^{20}$ Lastly, we introduced another compartment representing the vaccinated individuals under the assumption that once vaccinated the individual will not be susceptible and will not be infected in the 
Flowchart 1: Schematic diagram of model architecture. A schematic diagram representing the model architecture and compartments is shown. Transitions between two compartments are shown as lines and arrows. Immunization is only considered at birth, and adult vaccination against hepatitis B virus (HBV) has not been included. From live birth compartment, transition can occur at multiple levels. For instance, at birth a child can be immunized that thus will not go into the susceptible compartment. Alternatively, if the child has not been vaccinated at birth, it can undergo a transition to either susceptible (if born to an HBVinfected mother) or healthy (if born to a non-HBV-infected mother). All the compartments can potentially be transitioned to death compartment

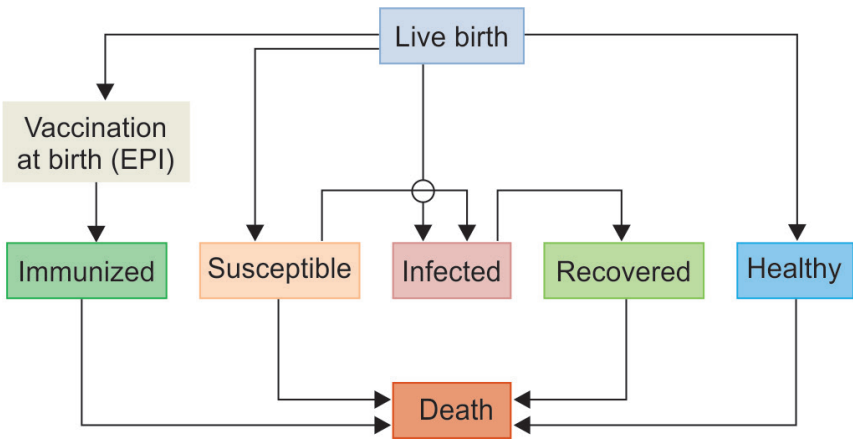

future due to long-lasting immunity. A schematic figure describing the compartmentalization of the model is shown in Flowchart 1. For vaccination, only vaccination at birth was considered and adult vaccination was ignored.

\section{Equations}

The following equations were developed to describe the dynamics of the model, where $\mathrm{d} S / \mathrm{d} t, \mathrm{~d} l / \mathrm{d} t$, and $\mathrm{d} R / \mathrm{d} t$ represent the dynamics of susceptible, infected, and recovered population over time.

$$
\frac{\mathrm{d} S}{\mathrm{~d} t}=\alpha s B+v s l-\mu s S-\beta S-v S
$$

$$
\begin{gathered}
\frac{\mathrm{d} l}{\mathrm{~d} t}=\alpha i B-v s I-v r l-\mu i l+\beta S \\
\frac{\mathrm{d} R}{\mathrm{~d} t}=\alpha r R+v r l+v S-\mu r R
\end{gathered}
$$

Where

- S, I, and $R$ correspond to susceptible, infected, and recovered population

- B corresponds to birth population

- $\alpha s, \alpha i$, and $\alpha r$ correspond to the proportion of population that are susceptible, infected, and recovered at birth

- $\quad v S$ and $v R$ correspond to the proportion of infected population that become susceptible and recovered, respectively

- $B$ and $v$ correspond to the proportion of susceptible population that become infected and recovered, respectively

- $\mu s, \mu i$, and $\mu r$ correspond to the proportion of susceptible, infected, and recovered state that die

\section{Assumptions}

- $\alpha s+\alpha i+\alpha r=1$

- None can move from susceptible or infected state from recovered state;

- Once recovered, they stay recovered

- Infected population cannot become susceptible once again, therefore, $v S$ should be 0 .

- The effect of vaccination can be modeled by increasing the values of $v R$ and $v S$ compared to "no vaccination rate."

\section{Model Parameters}

The following parameters were used for model simulation. Some parameters were retrieved from the existing literature and the World Health Organization (WHO) statistical fact sheets. Table 1

\begin{tabular}{|c|c|c|}
\hline Parameters & Value & Reference \\
\hline Birth rate & 2.14 per woman & $\begin{array}{l}\text { World bank: https://data.worldbank.org/indicator/SP.DYN. } \\
\text { TFRT.IN?locations=BD }\end{array}$ \\
\hline Adult mortality rate & 5.40 deaths $/ 1,000$ population & $\begin{array}{l}\text { World Health Organization (WHO) fact sheet } 2016 . \\
\text { (https://www.who.int/countries/bgd/en/) }\end{array}$ \\
\hline Maternal mortality rate & 176 deaths/100,000 population & $\begin{array}{l}\text { World Health Organization (WHO) fact sheet } 2016 . \\
\text { (https://www.who.int/countries/bgd/en/) }\end{array}$ \\
\hline Neonatal mortality rate at birth & 23.3 deaths $/ 1,000$ population & $\begin{array}{l}\text { World Health Organization (WHO) expanded program } \\
\text { on immunization (EPI) fact sheet 2016. http://www.searo. } \\
\text { who.int/immunization/data/bangladesh.pdf }\end{array}$ \\
\hline Infant ( $<1$ year) mortality rate & 30.7 deaths $/ 1,000$ population & $\begin{array}{l}\text { World Health Organization (WHO) expanded program } \\
\text { on immunization (EPI) fact sheet 2016. http://www.searo. } \\
\text { who.int/immunization/data/bangladesh.pdf }\end{array}$ \\
\hline Child ( $<5$ years) mortality & 37.6 deaths $/ 1,000$ population & $\begin{array}{l}\text { World Health Organization (WHO) expanded program } \\
\text { on immunization (EPI) fact sheet 2016. http://www.searo. } \\
\text { who.int/immunization/data/bangladesh.pdf }\end{array}$ \\
\hline Vaccination rate (HepB3) & $90 \%$ & $\begin{array}{l}\text { World Health Organization (WHO) expanded program } \\
\text { on immunization (EPI) fact sheet 2016. http://www.searo. } \\
\text { who.int/immunization/data/bangladesh.pdf }\end{array}$ \\
\hline True vaccination rate (HepB3) & $80 \%$ considering $10 \%$ dropout & $\begin{array}{l}\text { World Health Organization (WHO) expanded program } \\
\text { on immunization (EPI) fact sheet 2016. http://www.searo. } \\
\text { who.int/immunization/data/bangladesh.pdf }\end{array}$ \\
\hline
\end{tabular}
summarizes the parameter description, values, and references.

Table 1: Summary of model stimulation: parameters, values and references 
Apart from these demographic parameters, two other important parameters are infection rate at which a susceptible person is infected and recovery rate at which an infected person recovers. These two parameters were estimated based on extensive literature survey. For instance, Mahtab et al. reported that the prevalence of HVB in Bangladesh is $5.5 \%$ based on the analysis of the HBsAg status of 1,018 individuals. ${ }^{5}$ However, another study using the secondary data from 16 different sources concluded that the prevalence of HVB-infected individual is $3.1 \%{ }^{2}$ However, the prevalence data of $\mathrm{HBV}$ infection in children under the age of 5 are lacking. In order to employ the current model to investigate the effect of vaccination among children, we estimated the HBV infection rate among infants and children as 0.157 and 0.179 , respectively, based on the previous studies. ${ }^{21}$

\section{Results}

\section{Estimate HBV Infection Rate in Children up to 2030 by Dynamic Modeling}

First, we aim to estimate the HBV-induced infection rate in children below 5 years of age. The model simulations were performed up to the year 2030. For the simulation purpose, three different vaccination rate scenarios were considered, where the different vaccination coverage $(80,90$, and $95 \%)$ were included. Simulation revealed that the number of infected children is likely to increase up to year 2030 despite vaccination and irrespective of different vaccination rates compared to the initial number of infected children. Comparison of different vaccination rates showed that the number of HBV-infected children will be the lowest for the highest vaccination rate (95\%), followed by $90 \%$ and $80 \%$ vaccination rate as expected (Fig. 1). The highest infection rate was estimated for the year 2023, and this highest infection rate was set to $100 \%$. Subsequently for all other years the fraction of the infection rate with respect to the highest infection rate is shown in $Y$-axis (Fig. 1). For different vaccination rates, the initial number of HVB-infected children represents the children who are already being infected with $\mathrm{HBV}$ at the beginning of simulation. The incidence of HVB-induced infection rate in the year 2030 is likely to be around $90 \%$ for $80 \%$ vaccination rate. The percentage of infected children dramatically decreased (45\%) for $90 \%$ vaccination rate. The lowest infection rate $(22 \%)$ was observed for $95 \%$ vaccination rate. Interestingly, only $10 \%$ increase in the vaccination rate from 80 to $90 \%$ is likely to contribute to the significant lowering (around 40\%) of HBV-induced infection rate among children. When increased by $5 \%$ vaccination rate from 90 to $95 \%$, the HBV-infection rate can be further decreased by $22 \%$, underscoring the significant effect of vaccination in reducing HBV infection among children. However, the according to the SDG requirements, a $90 \%$ reduction in $\mathrm{CHB}$ infection (indicated by green dash line) is desired by the year 2030. Even the highest (95\%) vaccination rate failed to meet the SDG requirement by the year 2030 (Fig. 1).

\section{Hepatitis B Virus-induced Death Rate Estimation from 2050 Onward}

Next we investigated the impact of current vaccination on future HBV-induced death rate in adults. The year-by-year model simulation showed that the future HBV-induced death rates depend on the current vaccination rate. Three different vaccination rates $(80,90$, and $95 \%)$ were considered as previously described. The time frame was set from 2050 to 2100 due to simulation of the
HBV-induced death rates (Fig. 2). Three different vaccination rates from 2020 onward is likely to generate differential HBV-induced death rates in the future (from 2050 onward). This time lag from vaccination to $\mathrm{HBV}$ death rate estimation was introduced to simulate the real-life scenario under the assumption that a chronically infected child may develop HCC and be subjected to HBV-related death after the age of 40 as described by Wang et al. ${ }^{11}$ Expectedly, the highest and the lowest death rates are likely to occur for the lowest (80\%) and the highest (95\%) vaccination rates among the tested vaccination scenarios (Fig. 2). Unlike the HBV-infection rate, the initial HVB-induced death rate was set to zero to monitor the new HBV-induced death incidents from 2050 to 2100. The fraction (\%) of death rates was calculated with respect to this highest death rate that was observed in the year 2070 . Death rate is likely to be increased from 2060 compared to earlier time points, meaning that children, who are not vaccinated at 2020, can potentially develop chronic infection and are most susceptible to HBV-induced death by the year 2060 and onward. The average HVB-induced death rate (indicated as red dash line) from the year 2060 to 2100 is likely to be around $90 \%$ for $80 \%$ vaccination rate followed by $47 \%$ death rate for $90 \%$ vaccination (blue dash line). The lowest average death rate $(23 \%)$ is observed for $95 \%$ vaccination rate (green dash line). Interestingly, $45 \%$ reduction in $\mathrm{HBV}$-induced death can potentially be achieved by only $10 \%$ increase in the vaccination rate from 80 to $90 \%$. When increased by $5 \%$ vaccination rate from 90 to $95 \%$, the HBV-infection rate can potentially be decreased by $24 \%$, underscoring the significant effect of vaccination in reducing the

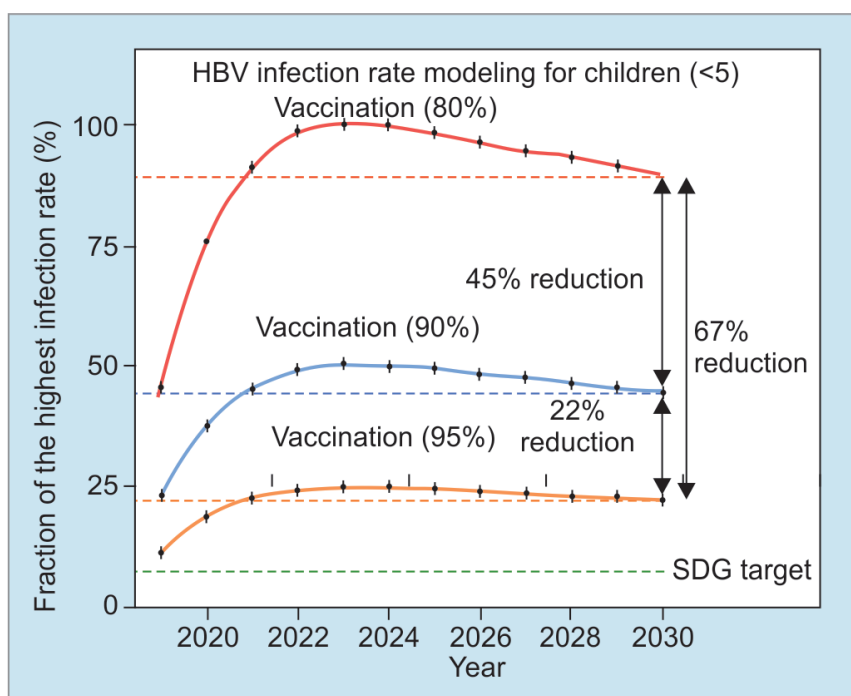

Fig. 1: Model simulation of dynamic hepatitis B virus (HBV)-induced infection rates at three different vaccination rates. Model simulation of HBV-induced infection rate among children ( $<5$ years) from 2020 to 2030 at three different vaccination rates are shown. Infection rates at vaccination rate of 80,90 , and $95 \%$ are shown as red, blue, and orange lines, respectively. The $Y$-axis represents the percentages as the fraction of the highest infection rate observed. For instance, the highest infection rate was observed at $80 \%$ vaccination rate for the year 2023 and thus this value was set to $100 \%$ in the $Y$-axis and subsequently all other infection rates are presented as a fraction of this highest infection rate. The horizontal dash lines represent the infection rate by the year 2030 for three different vaccination rate-80 (red dash line), 90 (blue dash line), and $95 \%$ (orange dash line). The green dash line represents the desired infection rate by 2030 per the SDG guidelines. The reduction in infection rates between the two different vaccination rates are indicated by vertical solid lines with arrows 


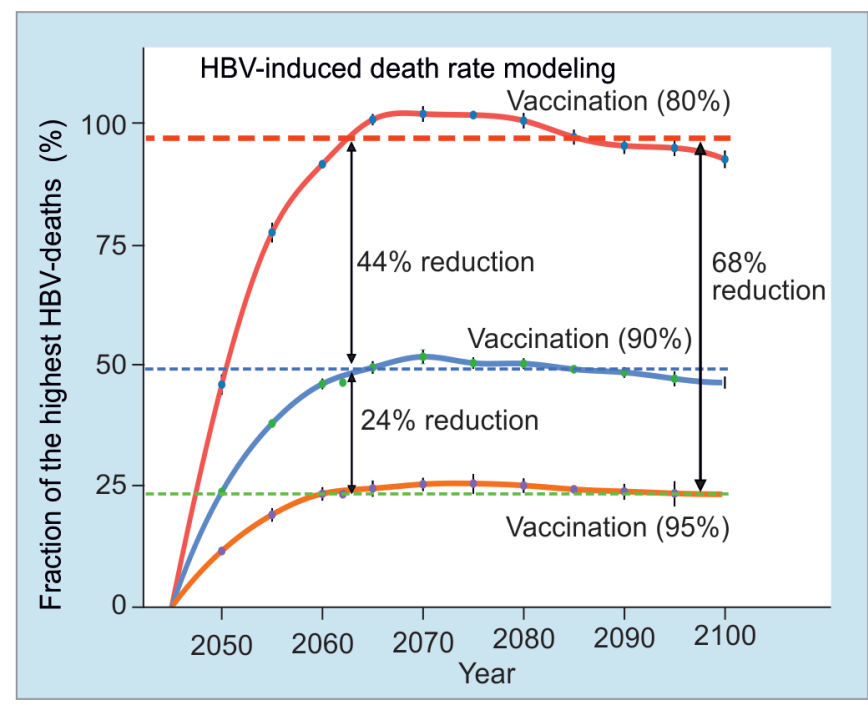

Fig. 2: Model simulation of dynamic hepatitis B virus (HBV)-induced death rates at three different vaccination rates. Model simulation of HBVinduced mortality (death) rate among children ( $<5$ years) from 2050 to 2100 at three different vaccination rates are shown. The HBV-induced death rates at vaccination rate of 80,90 , and $95 \%$ are shown as red, blue, and green lines, respectively. The $Y$-axis represents the percentages as the fraction of the highest death rate that were observed for the year 2070. This value was set to $100 \%$ in the $Y$-axis and subsequently all other death rates are presented as a fraction of this highest death rate. The horizontal dash lines represent the average infection rate for three different vaccination rates-80 (red dash line), 90 (blue dash line), and $95 \%$ (green dash line). The reduction in infection rates between two different vaccination rates are indicated by vertical solid lines with arrows

future mortality rate. In case of HB-induced death rate, to attain SDG, the WHO recommended to reduce the mortality by $65 \%$ which may not be achieved by 80 and $90 \%$ vaccination rates, rather vaccination rate of $95 \%$ has the potential to meet this challenge of reducing the required fraction of child mortality due to HBV infection.

\section{Gender-specific HBV Infection Rate in Children}

Having established the HBV-induced infection rate in children ( $<5$ years) of both sexes under the assumption that immunization is gender neutral, it implies that male and female children are equally vaccinated. Although the vaccination is largely supposed as gender neutral, it has been reported that negative biasness exists in vaccination coverage. ${ }^{22}$ According to GAVI "Gender and immunization abridged report" by Overseas Development Institute (www.odi.org.uk), there is a larger gender inequality which is exacerbated in the lower wealth quintiles of a population and in the majority of cases is directed against girls in Bangladesh. For instance, in Bangladesh, the ratio of girls to boys not immunized can be as low as 0.5 (bias against girls) in the poorest quintile. For the current study, the female to male vaccination coverage ratio of 0.8 was set on an average throughout all socioeconomic quintiles. We wanted to investigate the impact of this negative bias contributing to a differential infection rate in male and female children. When the female to male vaccination ratio of 0.8 was included into the model, the result showed a surprising difference in HBV infection in male and female children (Fig. 3). With $90 \%$ vaccination rate, the negative bias contributes

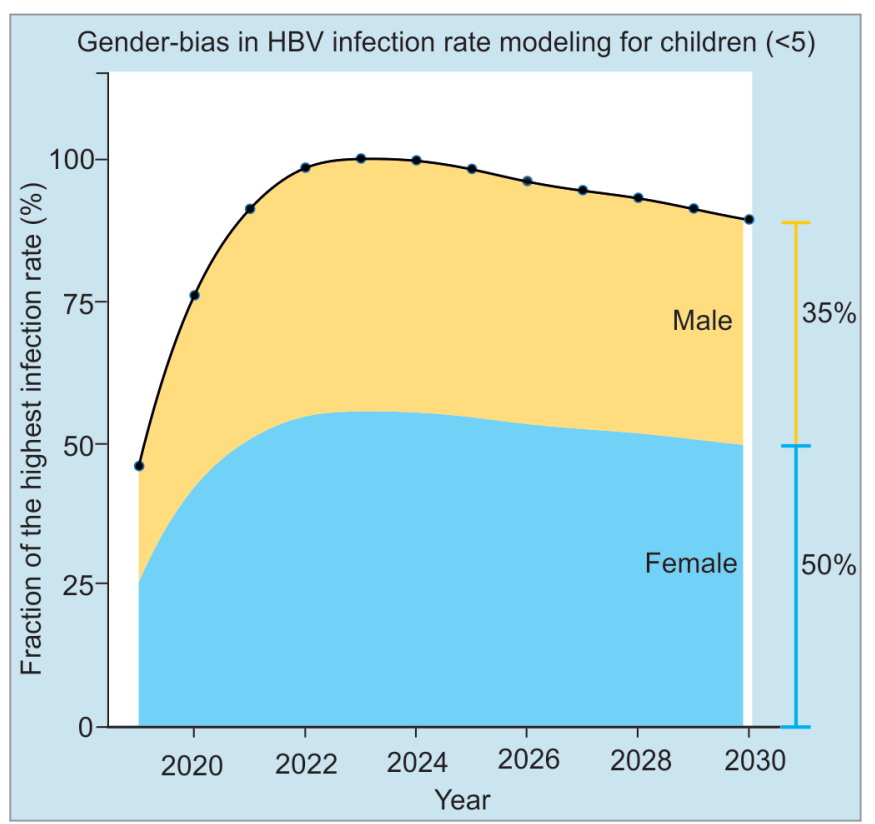

Fig. 3: Model simulation to determine the impact of negative bias on hepatitis B virus (HBV)-induced infection rates. A gender bias in immunization toward girls was implemented in the model by including the vaccination ratio of 0.8 (female to male children). Model simulation of HBV-induced infection rates among male and female children ( $<5$ years) is shown from 2020 to 2030 at $90 \%$ vaccination rate. The $Y$-axis represents the percentages as the fraction of the highest infection rate that were observed for the year 2023. This value was set to $100 \%$ in the $Y$-axis and subsequently all other infection rates are presented as a fraction of this highest infection rate. The black curve represents the dynamic year-to-year infection rate of all children including males and females. Area under the curves represents the total male (orange area) and female (blue area) children that are likely to be infected during the simulation period (from 2019 to 2030). The vertical lines indicate the percentage of male (orange) and female (blue) children that are likely to be infected by the year 2030

to 35 and $50 \%$ infection rate (as a fraction of highest infection rate) for male and female children, respectively. The $15 \%$ increase in HBV infection in female children is attributed by the one variable factor in the form of negative bias toward girls in the immunization program.

\section{Gender-specific HBV-induced Death in Children}

After observing the surprising increase in the HBV-infection rate in the female children due to negative bias in vaccination, we asked whether this biasness also influences the HBV-induced death rates in female population in the future. The simulation of HBV-induced death rates from 2050 to 2100 with $90 \%$ vaccination rate revealed that female subjects are more vulnerable to HBV-induced death compared to their male counterparts. The model simulation showed that at a vaccination rate of $90 \%$, negative bias toward girls may lead to an average HBV-induced death rate of $44 \%$ and $56 \%$ for male and female, respectively, thereby increasing the female death rate by $12 \%$ (Fig. 4).

\section{Discussion}

The current study revealed an alarming scenario about the HBVinduced infection and death rates in Bangladesh despite the 
Impact of negative-bias of immunization against girls on HBV-induced death rate in adult

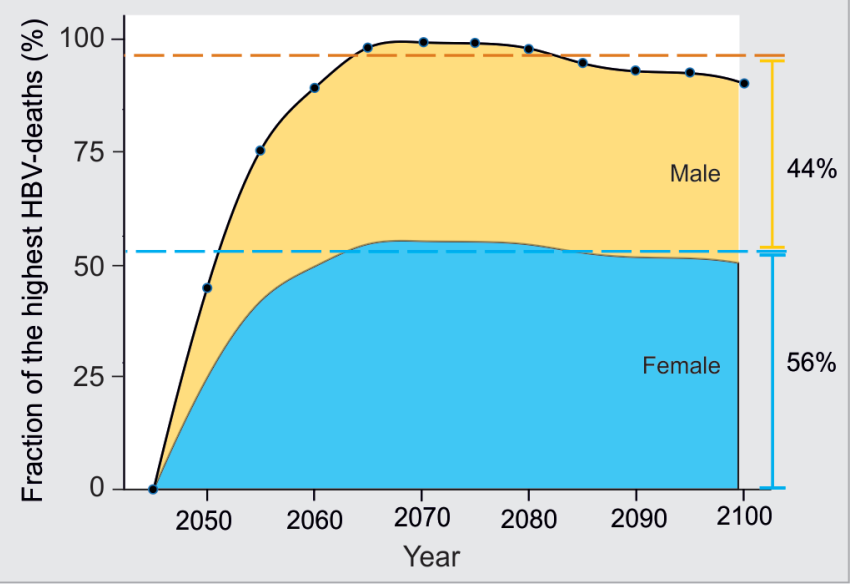

Fig. 4: Model simulation to determine the impact of negative bias on hepatitis B virus (HBV)-induced death rates. A gender bias in immunization toward girls was implemented as described in Figure 3. Model simulation of HBV-induced death rates among male and female children ( $<5$ years) is shown from 2019 to 2030 at $90 \%$ vaccination rate. The $Y$-axis represents the percentages as the fraction of the highest infection rate that were observed at 2024. This value was set to $100 \%$ in the $Y$-axis and subsequently all other death rates are presented as a fraction of this highest death rate. The black curve represents the dynamic year-to-year death rate of all subjects including males and females. Area under the curves represents the total male (orange area) and female (blue area) population that are likely to die due to HVB infection during the simulation period (from 2050 to 2100). The horizontal dash lines represent average death rates for male (orange) and female (blue) populations. The vertical lines indicates the percentage of male (orange) and female (blue) children that are likely to be subjected to HBV-induced mortality from 2050 to 2100

current ongoing vaccination efforts under the EPI. Generally, EPI in Bangladesh represents a success story where the EPI was able to achieve high vaccination coverage against vaccinepreventable diseases. ${ }^{15}$ However, the caveats in estimation of EPI coverage cannot be ignored in order to attain SDG by the year 2030. For example, earlier study showed that the estimation of vaccination coverage is incomplete and often includes a number of vaccinated children of specified age range (12-23 months) only, and the authors argued that it lacks the information of how many children attended the scheduled vaccination. ${ }^{23}$ In a recent study, Sheikh et al. identified that the fraction of children receiving scheduled vaccination is much lower than anticipated. ${ }^{15}$ For instance, only 24,46 , and $53 \%$ of children were able to receive timely vaccination for BCG, pentavalent-3, including HBV-vaccine and measles, respectively. ${ }^{15}$ On the contrary, a relatively high percentage of children-76, 51, and $36 \%$ were unable to receive the $B C G$, pentavalent 3, and measles vaccines, respectively, in the recommend scheduled dates. ${ }^{15}$ Several factors that may influence the failure to vaccinate children in the recommended schedules were identified. Among these factors the most significant ones include maternal education coupled with their working status, effectiveness of the community awareness program, socioeconomic status, and the cultural practice depending on geographic locations. ${ }^{15}$ Apart from the failure to vaccinate children in a timely manner, another impeding challenge is dropouts from vaccination program that may render incomplete immunity against
HBV infection and may pose a challenge to obtain the desired reduction in $\mathrm{HBV}$-induced infection and death rates in Bangladesh. A study conducted by Khan et al. reported that the dropout rate can be as high as $13 \%$ for EPI in the urban area of Dhaka city among the lower income population. ${ }^{24}$ In conclusion, the authors argued that the current EPI, although has high coverage in many areas, increased the rates of dropouts and invalid doses threatens to reduce these numbers of fully immunized child to $66 \% .{ }^{24}$ In 2018 , WHO published the EPI fact sheet where over $80 \%$ coverage for DTPHib-HepB3 vaccine with greater than 10\% dropout (http://www. searo.who.int/immunization/data/fact_sheets/en/) was reported. Quaiyum et al. systematically identified the multiple factors that alone or in combination may lead to the higher dropout rates. ${ }^{25}$ The most important among these factors were irregular EPI sessions, followed by no notification system regarding the subsequent session/doses, uncooperative behavior of vaccine-care providers, nonappearance of vaccine provider, poor documentation and consequently refusing to vaccinate due to the loss of record. ${ }^{25}$ The combination of untimely vaccination coupled with vaccination dropouts reduces the total vaccination coverage. We performed simulations with three different vaccination rates: 80,90 , and $95 \%$. According to the WHO guidelines, $\mathrm{HBV}$-induced infection and death rates should be reduced by $90 \%$ and $65 \%$, respectively, by the year 2030 (https://www.who.int/hepatitis/publications/hepelimination-by-2030-brief/en/). In these circumstances, our model simulation revealed a distressing scenario as far as the reduction in the HBV-induced infection and death rates are concerned. The model analysis showed that even if we take adequate measures to ensure timely vaccination and prevent dropouts and increase the vaccination rate up to $95 \%$, still will be unable to reduce HBVinfection rate by $90 \%$ by the year 2030 . On the contrary, reduction in $\mathrm{HB}$-induced death rate by $65 \%$ in the future may not be achievable by $80 \%$ and $90 \%$ vaccination rate. Hence, we must take adequate steps to increase vaccination rates to $95 \%$ to reduce the HBVinduced child mortality to attain SDG.

The next challenge in the vaccination is the negative bias toward girls. According to GAVI Alliance report (https://www.odi.org/ publications/4421-gender-and-immunisation-abridged-report), there is a tendency that female children often have limited access to follow-up vaccination services compared to their male counterparts, implying that a preferential allocation of vaccine-related resources to male children is happening in Bangladesh. In Bangladesh, female children are often deprived of vaccination follow-up services especially in lower socioeconomic quintiles. We aimed to investigate the impact of negative bias in vaccination toward newborn girls by aiming female to male vaccination coverage ratio at 0.8 as reported by GAVI Alliance. This difference may seem to be insignificant; however, the simulation of HBV-induced infection and death rates surprisingly uncovered how this apparently small negative bias in vaccination may result in a large difference between girls and boys by the year 2030 . Results revealed that at $90 \%$ vaccination rate, the negative bias can potentially contribute a $15 \%$ increase in HBV-induced infection that subsequently may lead to $12 \%$ increase in HBV-induced mortality rate in adult females in the future. This increase in HBV infection and HBV-induced death rates in female population is likely to have a far reaching effect on the population level. The increased number of girls infected with HBV either through mother-to-child vertical transfer or through other blood-borne route may develop chronic infection and when these infected girls reach child-bearing age, they may transfer the HBV to newborn children during birth. Therefore, to reduce HBV-induced infection 
and death rates, it is of utmost importance to prevent the negative bias of immunization toward girls.

\section{Conclusion}

In conclusion, the current study aided by mathematical modeling unearths the alarming possibility of increased HBV-induced infection especially in girls by the year 2030. These increased rates of infection will eventually intensify the HBV-induced death rates in the future time in Bangladesh, posing a grand challenge to attain SDG. Therefore, in light of the mathematical model, we propose several proposals including ensuring timely and full completion of the pentavalent vaccination program, reducing dropouts from vaccination program, and lastly preventing negative bias toward girls during the immunization program.

\section{Acknowledgment}

We acknowledge the Department of Hepatology, BSMMU for the support and providing data for model calibration.

\section{References}

1. Liang TJ. Hepatitis B: the virus and disease. Hepatology 2009; 49(5 Suppl):S13-S21. DOI: 10.1002/hep.22881.

2. Schweitzer A, Horn J, Mikolajczyk RT, et al. Estimations of worldwide prevalence of chronic hepatitis $B$ virus infection: a systematic review of data published between 1965 and 2013. Lancet 2015;386(10003):1546-1555. DOI: 10.1016/S0140-6736(15)61412-X.

3. Ott JJ, Stevens GA, Groeger J, et al. Global epidemiology of hepatitis B virus infection: new estimates of age-specific $\mathrm{HBsAg}$ seroprevalence and endemicity. Vaccine 2012;30(12):2212-2219. DOI: 10.1016/ j.vaccine.2011.12.116.

4. Wait $S$, Kell E, Hamid S, et al. Hepatitis $B$ and hepatitis $C$ in southeast and southern Asia: challenges for governments. Lancet Gastroenterol Hepatol 2016;1(3):248-255. DOI: 10.1016/S2468-1253(16) 30031-0.

5. Mahtab MA, Rahman S, Karim MF, et al. Epidemiology of hepatitis B virus in Bangladeshi general population. Hepatobiliary Pancreat Dis Int 2008;7(6):595-600.

6. Franco E, Bagnato B, Marino MG, et al. Hepatitis B: epidemiology and prevention in developing countries. World J Hepatol 2012;4(3):74-80. DOI: 10.4254/wjh.v4.i3.74.

7. Zanetti AR, Van Damme P, Shouval D. The global impact of vaccination against hepatitis B: a historical overview. Vaccine 2008;26(49): 6266-6273. DOI: 10.1016/j.vaccine.2008.09.056.

8. Borgia G, Carleo MA, Gaeta GB, et al. Hepatitis B in pregnancy. World J Gastroenterol 2012;18(34):4677-4683. DOI: 10.3748/wjg.v18.i34. 4677.

9. Di Bisceglie AM. Hepatitis B and hepatocellular carcinoma. Hepatology 2009;49(5 Suppl):S56-S60. DOI: 10.1002/hep.22962.
10. Bixler $\mathrm{D}$, Zhong $\mathrm{Y}$, Ly KN, et al. Mortality among patients with chronic hepatitis B infection: the chronic hepatitis cohort study (CHeCS). Clin Infect Dis 2019;68(6):956-963. DOI: 10.1093/cid/ciy598.

11. Wang T. Model of life expectancy of chronic hepatitis B carriers in an endemic region. J Epidemiol 2009;19(6):311-318. DOI: 10.2188/jea. JE20090039.

12. Meireles LC, Marinho RT, Van Damme P. Three decades of hepatitis B control with vaccination. World J Hepatol 2015;7(18):2127-2132. DOI: 10.4254/wjh.v7.i18.2127.

13. WHO Publication. Hepatitis B vaccines: WHO position paperrecommendations. Vaccine 2010;28(3):589-590. DOI: 10.1016/ j.vaccine.2009.10.110.

14. Paul RC, Rahman M, Wiesen E, et al. Hepatitis B surface antigen seroprevalence among prevaccine and vaccine era children in Bangladesh. Am J Trop Med Hyg 2018;99(3):764-771. DOI: 10.4269/ ajtmh.17-0721.

15. Sheikh N, Sultana M, Ali N, et al. Coverage, timelines, and determinants of incomplete immunization in Bangladesh. Trop Med Infect Dis 2018;3(3):E72. DOI: 10.3390/tropicalmed3030072.

16. Boulton ML, Carlson BF, Power LE, et al. Socioeconomic factors associated with full childhood vaccination in Bangladesh, 2014. Int J Infect Dis 2018;69:35-40. DOI: 10.1016/j.ijid.2018.01.035.

17. Omori R, Cowling BJ, Nishiura H. How is vaccine effectiveness scaled by the transmission dynamics of interacting pathogen strains with cross-protective immunity? PLoS One 2012;7(11):e50751. DOI: 10.1371/ journal.pone.0050751.

18. Baussano I, Franceschi S, Plummer M. Infection transmission and chronic disease models in the study of infection-associated cancers. Br J Cancer 2014;110(1):7-11. DOI: 10.1038/bjc.2013.740.

19. Morris SE, Pitzer VE, Viboud C, et al. Demographic buffering: titrating the effects of birth rate and imperfect immunity on epidemic dynamics. J R Soc Interface 2015;12(104):20141245. DOI: 10.1098/ rsif.2014.1245.

20. Navabakhsh B, Mehrabi N, Estakhri A, et al. Hepatitis B virus infection during pregnancy: transmission and prevention. Middle East J Dig Dis 2011;3(2):92-102.

21. Uz-Zaman MH, Rahman A, Yasmin M. Epidemiology of hepatitis B virus infection in Bangladesh: prevalence among general population, risk groups and genotype distribution. Genes (Basel) 2018;9(11):E541. DOI: 10.3390/genes9110541.

22. Merten S, Martin Hilber A, Biaggi C, et al. Gender determinants of vaccination status in children: evidence from a meta-ethnographic systematic review. PLoS One 2015;10(8):e0135222. DOI: 10.1371/ journal.pone.0135222.

23. Salmon DA, Dudley MZ, Glanz JM, et al. Vaccine hesitancy: causes, consequences, and a call to action. Vaccine 2015;33(Suppl 4): D66-D71. DOI: 10.1016/j.vaccine.2015.09.035.

24. Khan MN, Rahman ML, Awal Miah A, et al. Vaccination coverage survey in Dhaka district. Bangladesh Med Res Counc Bull 2005;31(2):46-53.

25. Quaiyum MA, Gazi R, Khan Al, et al. Programmatic aspects of dropouts in child vaccination in Bangladesh: findings from a prospective study. Asia Pac J Public Health 2011;23(2):141-150. DOI: 10.1177/1010539509342119. 